\title{
Response to BNT162b2 mRNA COVID-19 vaccine among healthcare workers in Italy: a 3-month follow-up
}

\author{
Domenico Ponticelli ${ }^{1} \cdot$ Fabiana Madotto $^{2} \cdot$ Sara Conti $^{3} \cdot$ Ippazio C. Antonazzo $^{3} \cdot$ Andrea Vitale $^{1}$. \\ Giovanni Della Ragione ${ }^{1}$. Maria L. Romano ${ }^{1}$ - Mario Borrelli ${ }^{1}$ Beniamino Schiavone ${ }^{1} \cdot$ Riccardo Polosa $^{4,5}$. \\ Pietro Ferrara ${ }^{1,2,3,6}$ (D) Lorenzo G. Mantovani ${ }^{2,3}$
}

Received: 9 July 2021 / Accepted: 21 September 2021 / Published online: 12 October 2021

(c) The Author(s) 2021

\begin{abstract}
This study investigated the response to BNT162b2 mRNA COVID-19 vaccine among healthcare workers (HCWs) in an Italian teaching hospital. 444 participants were surveyed with either multiple RT-PCR assays for detection of SARS-CoV-2 nucleic acid in nasopharyngeal swabs or serology testing for the research of virus-specific immunoglobulins. Adverse events following immunization (AEFI) were reported. Two weeks after the first dose anti-SARS-CoV-2 antibodies exceeded reactivity cut-off in $82.5 \%$ the participants. Four HCWs tested positive at nasopharyngeal swab after 3 months. More than threequarters reported AEFIs. Our findings offer an insight regarding the vaccine response after 3 months from its administration, with a special focus on effectiveness data, as well as the type and number of AEFIs complained by HCW recipients. The presented study may serve as reference for future research which will be necessary to explore the long-term safety of this vaccine, especially in population at high risk for infection, such as HCWs.
\end{abstract}

Keywords BNT162b2 vaccine $\cdot$ COVID-19 $\cdot$ Healthcare workers $\cdot$ SARS-CoV-2

\section{Introduction}

Effective and timely immunologic response to vaccines is a crucial strategy for the control of the severe acute respiratory syndrome-coronavirus 2 (SARS-CoV-2) pandemic. The two-dose messenger RNA (mRNA) vaccine BNT162b2 showed an efficacy of $94.8 \%$ against novel coronavirus

Fabiana Madotto, Sara Conti and Ippazio C. Antonazzo have equally contributed to this work.

Pietro Ferrara

p.ferrara5@campus.unimib.it

1 Pineta Grande Hospital, Castel Volturno, Caserta, Italy

2 Value-Based Healthcare Unit, IRCCS MultiMedica, Sesto San Giovanni, Milan, Italy

3 Center for Public Health Research, University of Milan-Bicocca, Via Cadore 48, 20900 Monza, Italy

4 Center of Excellence for the Acceleration of HArm Reduction (CoEHAR), University of Catania, Catania, Italy

5 Department of Clinical and Experimental Medicine, University of Catania, Catania, Italy

6 Health Direction, MultiMedica Group, Milan, Italy disease 2019 (COVID-19). Its immune response is direct against the SARS-CoV-2 S1 spike protein, and antibody titers are associated with functional virus neutralization [1].

After a positive assessment of safety and efficacy for BNT 162b2 mRNA vaccine granted by different national and international regulatory agencies, a global vaccination campaigns targeted healthcare workers (HCWs) as the first vaccinees, both for ensuring their protection and safety at work, and the sustainability of healthcare systems [2,3]. Growing evidence is emerging about the vaccine response, but data on effectiveness and safety after the second dose deserve careful evaluation [4]. In particular, real-world accurate information regarding adverse events following immunization (AEFI) is important to educate the population and to foster vaccination campaign itself. Therefore, we assessed the response to BNT162b2 mRNA COVID-19 vaccine in a sample of HCWs of an Italian teaching hospital. 


\section{Methods}

Here, we present the results of a 3-month longitudinal observational study conducted among the healthcare workers (HCWs) of the Pineta Grande Hospital (Castel Volturno, Caserta, Italy) with the objective of evaluating the response to the two-dose mRNA vaccine BNT162b2 administered between December 2020 and January 2021.

All the internal HCWs were invited to participate in the study before the administration of the vaccine, performed according to the manufacturer's instruction as a two-dose regimen with an interval of 3 weeks. The participation was voluntary, vaccinees were not offered any incentive, and were informed about their right to withdraw at any time without penalty. The vaccinees were interviewed about their demographics and professional characteristics (sex, age, professional role, hospital unit, history of symptoms compatible with COVID-19, previous laboratory-confirmed SARS-CoV-2 infection). They were also asked to report any local (pain, redness, and swelling injection site) and systemic AEFI (fatigue, headache, muscle and joint pain, chills, fever, swelling of the face, tongue, throat, breathing problems) occurred within 7 days after the prime and booster doses. According to the study protocol, vaccinees were randomly divided in two groups, to undergo, respectively, (A) six nasopharyngeal swabs and real-time polymerase chain reaction (RT-PCR) assays for qualitative detection of SARS-CoV-2 nucleic acid; (B) or quantitative serology testing for the research of virus-specific immunoglobulins (Ig). The first tests were taken before the administration of the vaccine $\left(\mathrm{t}_{0}\right)$ and then according to a predefined timeline (at 15, 30, 45, 60, and 90 days from the first dose). Seroprevalence was assessed ADVIA Centaur ${ }^{\circledR}$ XPT Immunoassay System for the research of SARS-CoV-2 antibodies (Siemens Healthcare $\mathrm{GmbH}$, Munich, Germany) in serum samples, and reactivity was intended as antibody level Index $\geq 1.0$, according to manufacturer's instructions. Study protocol included up to six longitudinal serological tests, but tests were stopped upon the achievement of the maximum Index value of 10 .

SARS-CoV-2 infection was traced through RT-PCR on samples obtained from nasopharyngeal swabs, according to manufacturer's instructions. A passive surveillance follow-up for potential SARS-CoV-2 infections was set among the vaccinees after the end of the study.

A descriptive analysis was carried out to describe cohort characteristics and outcomes. Continuous variables were expressed as mean and standard deviation (SD), or median and interquartile range (IQR), according to their distribution. Categorical variables were described as absolute and relative frequency. Multivariate logistic regression analyses were built to investigate the association between the probability of seroconversion and AEFIs occurrence, and potential predictors, namely sex, age, and previous SARS-CoV-2 infection. Results were reported as adjusted odds ratios $\left(\mathrm{OR}_{\mathrm{adj}}\right)$ and $95 \%$ confidence intervals $(\mathrm{CI})$. All statistical tests were two tailed and a $p$ value $\leq 0.05$ was considered statistically significant.

\section{Results}

Overall, $444 \mathrm{HCWs}$ participated in this study: the characteristics of the study participants and their distribution into the two groups is presented in Table 1.

In the cohort A, six subjects tested positive at nasopharyngeal swabs within 15 days after the first vaccine dose and one about a week from the second dose. At 45- and 60-day follow-ups all vaccinees tested negative, but four positive tests were registered at the third month. Overall, two were asymptomatic and nine mildly symptomatic infections. In the cohort B, at 2 weeks after the first vaccine dose anti-SARS-CoV-2 antibodies exceeded the reactivity cutoff in $82.5 \%$ of the participants. At one-month follow-up, almost all (98.4\%) the vaccinees had reached the maximum Index value of 10. The likelihood of reaching this level at two-week follow-up was higher in younger HCWs $\left(\mathrm{OR}_{\mathrm{adj}}\right.$ 0.95 per annum; $95 \%$ CI $0.91-0.99 ; p=0.03$ ) and in those who had a baseline antibody level greater than the reactivity threshold $\left(\mathrm{OR}_{\mathrm{adj}} 13.18 ; 95 \%\right.$ CI $\left.3.30-52.71 ; p<0.001\right)$. In the whole sample $(n=444)$, one more case was reported to the in-hospital passive surveillance system in the third month after the end of the study.

AEFIs were studied in the whole sample. $75.5 \%$ and $80.0 \%$ the participants self-reported at least one AEFI after the first and the second dose, respectively. The most frequent AEFIs were local reactions at injection site. Among the participants, no life-threatening AEFIs were reported. No hospitalizations or visits to emergency department were observed in the study period. A complete AEFIs overview is reported in Table 2: they were found to be heterogeneously associated with sex and age. Fatigue, headache, muscle and joint pain, and fever after the first dose were more likely to be reported by subjects with a prior SARS-CoV-2 infection $\left(\mathrm{OR}_{\text {adj }} 2.19\right.$; 95\% CI 1.12-4.28; $p=0.02$ ). No significant association was found between the occurrence of any AEFIs at second dose and SARS-CoV-2 infection.

\section{Discussion}

Real-world data from this study offer an interesting insight regarding the vaccine response after 3 months from the administration with BNT162b2 vaccine. $82.5 \%$ the participants showed clinical markers of immunologic response 
Table 1 Study population

\begin{tabular}{|c|c|c|c|}
\hline \multicolumn{3}{|l|}{ Study population } & $N(\%)$ \\
\hline \multicolumn{3}{|l|}{ Total vaccinees } & 444 \\
\hline \multicolumn{3}{|l|}{ Age, mean $\pm \mathrm{SD}$} & $37.9 \pm 12.1$ \\
\hline \multicolumn{3}{|l|}{ Sex (male) } & $197(44.4)$ \\
\hline \multicolumn{3}{|l|}{ Previous SARS-CoV-2 infection } & $43(9.7)$ \\
\hline \multicolumn{3}{|l|}{ In close contact with patients } & $350(78.8)$ \\
\hline \multicolumn{4}{|l|}{ Healthcare workers } \\
\hline \multicolumn{2}{|l|}{ Physician } & & $54(12.2)$ \\
\hline \multicolumn{2}{|l|}{ Nurse } & & $197(44.4)$ \\
\hline \multicolumn{2}{|l|}{ Other HCWs } & & $29(6.5)$ \\
\hline \multicolumn{2}{|l|}{ Students } & & $74(16.7)$ \\
\hline \multicolumn{2}{|l|}{ Others } & & $90(20.3)$ \\
\hline \multicolumn{4}{|l|}{ Ward } \\
\hline \multicolumn{2}{|l|}{ Medical } & & $86(19.4)$ \\
\hline \multicolumn{2}{|l|}{ Surgical } & & $114(25.7)$ \\
\hline \multicolumn{2}{|l|}{ Other medical } & & $70(15.8)$ \\
\hline \multicolumn{2}{|l|}{ Nurse students } & & $74(16.7)$ \\
\hline \multicolumn{2}{|l|}{ Administrative } & & $100(22.5)$ \\
\hline \multicolumn{2}{|l|}{$\begin{array}{l}\text { Cohort of subjects who underwent blood test for detect- } N(\%) \\
\text { ing SARS-CoV-2 antibodies }\end{array}$} & Cohort of subjects who underwent SARS-CoV-2 swab & $N(\%)$ \\
\hline \multicolumn{2}{|l|}{ Cohort } & Cohort & 318 \\
\hline \multicolumn{2}{|l|}{ Age, mean $\pm \mathrm{SD}$} & Age, mean $\pm \mathrm{SD}$ & $36.9 \pm 12.3$ \\
\hline \multicolumn{2}{|l|}{ Sex (male) } & Sex (male) & $148(46.5)$ \\
\hline \multicolumn{2}{|l|}{ Previous SARS-CoV-2 infection } & Previous SARS-CoV-2 infection & $36(11.3)$ \\
\hline \multicolumn{2}{|l|}{ In close contact with patients } & In close contact with patients & $268(84.3)$ \\
\hline \multirow{3}{*}{\multicolumn{2}{|c|}{ Subjects with positive serological test at the enrollment 13 (10.3) }} & \multicolumn{2}{|l|}{$\begin{array}{l}\text { Time (days) between the first vaccine dose and swabs, median } \\
\text { [IQR] }\end{array}$} \\
\hline & & I swab & 15 [13-16] \\
\hline & & II swab & 30 [29-32] \\
\hline $\begin{array}{l}\text { Time (days) between 1st the first vaccine dose and blood } \\
\text { median [IQR] }\end{array}$ & serological tests, & III swab & $46[44-47]$ \\
\hline I blood test & $14[13-16]$ & IV swab & 60 [59-61] \\
\hline II blood test* & $30[29-31]$ & V swab & 91 [89-93] \\
\hline III blood test ${ }^{\circ}$ & $44[43-45]$ & Positive result to swab & \\
\hline Cumulative probability of reactivity $(\geq 1)$ & & I swab & $6(1.9)^{*}$ \\
\hline Time 1 & $104(82.5)$ & II swab & $1(0.3)^{*}$ \\
\hline Time 2 & $126(100)$ & III swab & $0(0.0)$ \\
\hline Time 3 & $126(100)$ & IV swab & $0(0.0)$ \\
\hline Cumulative probability of reactivity $(>10), n(\%)$ & & V swab & $4(1.3)^{*}$ \\
\hline Time 1 & $31(26.6)$ & & \\
\hline Time 2 & $124(98.4)$ & & \\
\hline Time 3 & $126(100)$ & & \\
\hline Antibody levels, median [IQR] & & & \\
\hline Time 1 & $2.9[1.2-9.6]$ & & \\
\hline Time 2 & $>10[>10->10]$ & & \\
\hline Time 3 & $>10[>10->10]$ & & \\
\hline
\end{tabular}

SARS-CoV-2 severe acute respiratory syndrome—coronavirus 2; $H C W$ healthcare worker; $S D$ standard deviation; IQR interquartile range

*All in close contact with patients 
Table 2 Adverse effects reported by $\mathrm{HCW}$ vaccinees $(n=444)$

\begin{tabular}{lll}
\hline Adverse effect & & \\
\hline After the first dose & & After the second dose \\
\hline $335(75.5)^{*}$ & At least one & $355(80.0)^{*}$ \\
$285(64.2)^{*}$ & Pain, redness, and swelling injection site & $271(61.0)^{*}$ \\
$168(37.8)^{*}$ & Fatigue & $248(55.9)^{*}$ \\
$126(28.4)^{* *}$ & Headache & $215(48.4)^{*}$ \\
$119(26.8)^{* *}$ & Muscle pain & $198(44.6)^{*}$ \\
$90(20.3)^{* *}$ & Chills & $173(39.0)^{* *}$ \\
$83(16.7)^{* *}$ & Joint pain & $148(33.3)$ \\
$25(5.63)$ & Fever & $111(25.0)^{*}$ \\
$8(1.8)$ & Enlarged lymph nodes & $26(5.9)$ \\
$2(0.5)$ & Urticaria & $4(0.9)$ \\
$7(1.6)$ & Swelling of face, tongue, or throat & $6(1.4)$ \\
$3(0.7)$ & Breathing problems & $2(0.5)$ \\
$11(2.5)$ & Others & $9(2.0)$ \\
\hline
\end{tabular}

*Association with sex (female) and age (younger age) $(p<.05)$

$* *$ Association with sex (female) $(p<.05)$ after the first BNT162b2 dose (second week) and 1 week after the second doses, almost all $(98.4 \%)$ the vaccinees developed anti-SARS-CoV-2 antibodies at the maximum Index value of 10. As expected, anti-S antibody level was boosted by the second dose, which consequentially increased the functional protection against SARS-CoV-2/COVID-19 $[1,5]$. More interestingly, we found that vaccine-induced antibody level was significantly high after 2 weeks from the single dose of SARS-CoV-2 mRNA vaccine in HCWs with preexisting immunity. Several data showed that one dose of the BNT162b2 vaccine (as well as other COVID-19 vaccines) maximizes the cellular and humoral immune response to $\mathrm{S} 1$ spike protein in subjects with previous SARS-CoV-2 infection and our results corroborate the hypothesis that previous SARS-CoV-2 infection may be considered an analogous to immune priming [6, 7].

COVID-19 cases have been reported in people who received one or both doses of the vaccine $[1,8,9]$. Four SARS-CoV-2 infections were registered at third month, with the development of a mild COVID-19-like symptomatology. Overall, the proportion of testing positive for SARS-CoV-2 after vaccination was $1.3 \%$, in line with a previous survey among HCWs $[4,9]$ but slightly higher than that registered among BNT162b2-trial participants. In particular, Keehner and coll highlighted important differences between the HCW populations and BNT162b2-trial participants, such as the availability of regular testing-irrespectively of symptoms' occurrence-and the younger age of the HCWs compared with the trial participants $[1,9]$. It should be also remarked the higher risk of SARS-CoV-2 exposure for hospital workers compared with the general population [2,3]. In addition, it is possible that those individuals with a PCR-positive swab before day 15 may not have already developed an efficacious immune response. In this regard, a study investigating the initial impact of SARS-CoV-2 vaccination on HCWs in Italy found that the reduction in the proportion of cases was only observed starting from around 30 days after the start of the vaccination campaign [4], likely depending on neutralizing antibody titers during the peri-infection period [10]. Interestingly, in the trimester after the end of the study period, only one SARS-CoV-2 case was registered at the surveillance follow-up.

As regards AEFIs occurrence, almost all the referred events were mild symptoms, with a vast majority of common local reactions to vaccines (pain, redness, or swelling injection site). No AEFIs other than the expected were observed, with a few suspected allergic reactions, confirming the safety profile of the BNT162b2 COVID-19 vaccine. Interestingly, the findings from this study showed that sex and age could affect AEFIs, with a higher likelihood in women and younger vaccinees. Evidence on sex-based differences in vaccine response has been previously described. Women showed a stronger immune response to vaccines that might enhance their effectiveness. On the other hand, this more effective innate and adaptive immune response might make women also more prone to experience AEFIs following vaccines administration [11]. Although a possible explanation of this phenomenon has been proposed, more sex-disaggregated data on COVID-19 vaccines are needed in order to deeply understand these sex differences. Similarly, younger age is associated to more a robust immune response, which could explain the higher AEs incidence compared with older recipients [12].

Some first-dose AEFIs-namely, fatigue, headache, muscle and joint pain, and fever-were associated with previous reports of laboratory-confirmed COVID-19, but 
at the second administration there was no difference in AEFIs occurrence between these recipients and those with no history of COVID-19. Larger studies should investigate duration and severity of AEFIs following mRNA vaccination in association with previous SARS-CoV-2 infection [6-9].

It is important to point out the main limitations of the presented study. The type of immunoassay system used for the research of SARS-CoV-2 antibodies in blood samples did not allow the study of the time trend of antibody levels in vaccine recipients; however, this was not intended as research objective. Again, although the most reported AEFIs are generally associated with vaccine administration, the presence of potential notoriety bias (defined as "a selection bias in which a case has a greater chance of being reported if the subject is exposed to the studied factor known to cause, thought to cause, or likely to cause the event of interest" [13]), with consequent over-reporting of certain AEFIs, cannot be completely excluded.

Despite these limitations, this research adds useful data to the current growing evidence on response to and safety of COVID-19 vaccines. It also offers direct practical implications that could contribute to refine the planning for future boosters to keep people protected. Lastly, this study focusses on AEFI incidence, offering important information that may refine current estimates of AEFI data and could positively contribute to vaccination acceptance.

In conclusion, this research adds important real-world data on the ongoing vaccination campaigns against COVID-19, the most valuable effective and safe strategy to protect the population against the disease. The low number of SARS-CoV-2 infections 3 months after administration of the second dose of vaccine is an important confirmation of the effectiveness of BNT162b2 mRNA COVID-19 vaccine, suggesting that it is maintained over time and among subjects at high risk for the infection. Longer follow-ups are needed to investigate the duration of the protection and time trends of antibody level, as well as to provide evidence-based metrics that may help maximize the current global vaccination campaign.

Acknowledgements This study is part of the VASCO project ("Monitoraggio della risposta al Vaccino Anti-Sars-cov-2/COvid-19 negli operatori sanitari del Pineta Grande Hospital") that studies the response to BNT162b2 mRNA COVID-19 vaccine among healthcare, in terms of effectiveness, immunogenicity, and safety. Dr. Ponticelli and Dr. Ferrara would like to thank Dr. Laura Spagnoli and Mr. Dario Albano for their logistical support.

Author contribution DP, and PF contributed to conceptualization; ICA, AV, GDR, and MLR were involved in data curation; FM, SC, and PF performed formal analysis; FM, SC, ICA, and PF were involved in methodology; PF was involved in project administration; FM and SC designed the software; RP, PF, and LGM performed supervision; DP, MB, BS, RP, PF, and LGM performed validation; LGM did visualization; PF was involved in writing—original draft; all the authors were involved in writing - review and editing.

Funding Open access funding provided by Università degli Studi di Milano - Bicocca within the CRUI-CARE Agreement. This research received no external funding.

Availability of data and material Data and supporting materials associated with this study will be provided upon request by contacting the corresponding author.

Code availability Not applicable.

\section{Declarations}

Conflict of interest RP is full tenured professor of Internal Medicine at the University of Catania (Italy) and Medical Director of the Institute for Internal Medicine and Clinical Immunology at the same University. In relation to his recent work in the area of respiratory diseases, clinical immunology, and tobacco control, RP has received has received lecture fees and research funding from Pfizer, GlaxoSmithKline, CV Therapeutics, NeuroSearch A/S, Sandoz, MSD, Boehringer Ingelheim, Novartis, Duska Therapeutics, and Forest Laboratories. Lecture fees from a number of European EC industry and trade associations (including FIVAPE in France and FIESEL in Italy) were directly donated to vaper advocacy no-profit organizations. RP has also received grants from European Commission initiatives (U-BIOPRED and AIRPROM) and from the Integral Rheumatology \& Immunology Specialists Network (IRIS) initiative. He has also served as a consultant for Pfizer, Global Health Alliance for treatment of tobacco dependence, CV Therapeutics, Boehringer Ingelheim, Novartis, Duska Therapeutics, ECITA (Electronic Cigarette Industry Trade Association, in the UK), Arbi Group Srl., and Health Diplomats. RP has served on the Medical and Scientific Advisory Board of Cordex Pharma, Inc., CV Therapeutics, Duska Therapeutics Inc, Pfizer, and PharmaCielo. RP is also founder of the Center for Tobacco prevention and treatment (CPCT) at the University of Catania and of the Center of Excellence for the acceleration of Harm Reduction (CoEHAR) at the same University, which has received support from Foundation for a Smoke Free World to conduct 8 independent investigator-initiated research projects on harm reduction. $\mathrm{RP}$ is also currently involved in the following pro bono activities: scientific advisor for LIAF, Lega Italiana Anti Fumo (Italian acronym for Italian Anti-Smoking League), the Consumer Advocates for Smoke-free Alternatives (CASAA), and the International Network of Nicotine Consumers Organizations (INNCO); Chair of the European Technical Committee for standardization on "Requirements and test methods for emissions of electronic cigarettes" (CEN/TC 437; WG4). L.G.M. received support from the Italian Ministry of Health Ricerca Corrente-IRCCS MultiMedica during the conduct of the study, and grants and personal fees from Bayer AG, Daiichi Sankyo, Pfizer, and Boehringer Ingelheim outside the submitted work. All other authors declare no conflict of interest.

Ethics approval This study was conducted according to the guidelines of the Declaration of Helsinki, and approved by the Institutional Review Board-Comitato Etico Campania Nord, with referral number CECN/1614/2021.

Informed consent All participants provided written informed consent before enrollment into the study, including agreeing for the results to be published.

Open Access This article is licensed under a Creative Commons Attribution 4.0 International License, which permits use, sharing, 
adaptation, distribution and reproduction in any medium or format, as long as you give appropriate credit to the original author(s) and the source, provide a link to the Creative Commons licence, and indicate if changes were made. The images or other third party material in this article are included in the article's Creative Commons licence, unless indicated otherwise in a credit line to the material. If material is not included in the article's Creative Commons licence and your intended use is not permitted by statutory regulation or exceeds the permitted use, you will need to obtain permission directly from the copyright holder. To view a copy of this licence, visit http://creativecommons. org/licenses/by/4.0/.

\section{References}

1. Polack FP, Thomas SJ, Kitchin N et al (2020) Safety and efficacy of the BNT162b2 mRNA COVID-19 vaccine. N Eng J Med 383:2603-2615

2. Della Valle P, Fabbri M, Madotto F et al (2021) Occupational exposure in the Lombardy region (Italy) to SARS-CoV-2 infection: results from the MUSTANG-OCCUPATION-COVID-19 study. Int J Environ Res Public Health 18(5):2567

3. Ferrara P, Albano L (2020) COVID-19 and healthcare systems: what should we do next? Public Health 185:1-2

4. Mateo-Urdiales A, Del Manso M, Andrianou X et al (2021) Initial impact of SARS-Cov-2 vaccination on healthcare workers in Italy-Update on the 28th of March 2021. Vaccine 39:4788-4792

5. Krammer F, Srivastava K, Alshammary H et al (2021) Antibody responses in seropositive persons after a single dose of SARSCoV-2 mRNA vaccine. N Eng J Med 384(14):1372-1374
6. Manisty C, Otter AD, Treibel TA et al (2021) Antibody response to first BNT162b2 dose in previously SARS-CoV-2-infected individuals. Lancet 397:1057-1058

7. Buonfrate D, Piubelli C, Gobbi F et al (2021) Antibody response induced by the BNT162b2 mRNA COVID-19 vaccine in a cohort of health-care workers, with or without prior SARS-CoV-2 infection: a prospective study. Clin Microbiol Infect. https://doi.org/10. 1016/j.cmi.2021.07.024

8. Dagan N, Barda N, Kepten E et al (2021) BNT162b2 mRNA Covid-19 vaccine in a nationwide mass vaccination setting. $\mathrm{N}$ Engl J Med 384:1412-1423

9. Keehner J, Horton LE, Pfeffer MA, et al. SARS-CoV-2 Infection after Vaccination in Health Care Workers in California N Engl J Med. 2021 May 6;384(18):1774-1775.

10 Bergwerk M, Gonen T, Lustig Y et al (2021) COVID-19 breakthrough infections in vaccinated health care workers. N Engl J Med. https://doi.org/10.1056/NEJMoa2109072 (Online ahead of print)

11. McCartney PR (2020) Sex-based vaccine response in the context of COVID-19. J Obstet Gynecol Neonatal Nurs 49(5):405-408

12. Jeon M, Kim J, Oh CE, Lee J (2021) Adverse events following immunization associated with coronavirus disease 2019 vaccination reported in the mobile vaccine adverse events reporting system. J Korean Med Sci 36(17):e114

13. Bégaud B, Dangoumau J (2000) Pharmacoepidemiology: definitions, problems, methodology. Therapie 55(1):113-117

Publisher's Note Springer Nature remains neutral with regard to jurisdictional claims in published maps and institutional affiliations. 